\title{
Professor Dr. Stephan Strobel, Dr.-Karl-Heyer-Preisträger 2002 - eine Laudatio
}

\author{
Mit dem Dr.-Karl-Heyer-Preis 2002 hat der Deutsche Allergie- und \\ Asthmabund den international angesehenen Pädiater und Aller- \\ gologen Prof. Dr. Stephan Strobel, London, ausgezeichnet. Der \\ Preisträger blickt auf eine jahrzehntelange Verbindung zur \\ deutschen Allergologie und speziell zur Deutschen Gesellschaft für \\ Allergologie und klinische Immunologie zurück, so dass an dieser \\ Stelle eine Würdigung erfolgen soll.
}

$\mathrm{T}$ raditionell wird auf dem Mönchengladbacher Allergie-Seminar des Deutschen Allergie- und Asthmabundes, organisiert und geleitet von Dr. Wolfgang Jorde, Mönchengladbach, der Dr.-Karl-Heyer-Preis verliehen in Erinnerung an den ehemaligen Chefarzt des Kamillianer-Krankenhauses, Mönchengladbach. Dr. Karl Heyer war Allergologe der ersten Stunde und eines der Gründungsmitglieder der Deutschen Gesellschaft für Allergologie und klinische Immunologie am 27. Oktober 1951 in Frankfurt am Main, damals als „Deutsche Allergiegesellschaft" eingetragen.

\section{Eine englisch-deutsche Karriere}

Der Preisträger des Jahres 2002, Prof. Dr. Stephan Strobel, geboren am 9. Oktober 1947, studierte in Frankfurt am Main von 1966-1972 und promovierte dort 1972 summa cum laude. Anschließend war er weiter in Frankfurt tätig, zunächst als Internist, dann als Pädiater bis zur Habilitation 1984. Er ging 1985 nach England, an das Department of Immunology des Institute of Child Health, London. Er hielt allerdings seine Verbindungen nach Deutschland über die Jahre hinweg aufrecht, wurde in Frankfurt 1992 apl. Professor und ist vielen von uns langjährig verbunden.

Seine zahlreichen beruflichen Schritte in Großbritannien können hier nicht alle erwähnt werden; es gehören hierzu die Funktion als Clinical Sub-
Dean seit 1992, die Professur des University College, London, 1996, die Funktion des Vice-Dean for Education and Training sowie des Deputy Head, Graduate School Biomedicine, UCL. Herr Strobel ist derzeit Professor of Paediatric and Clinical Immunology am University College London and Honorary Consultant in Clinical Immunology, Great Ormond Street Hospital for Children, NHS Trust, London.

\section{Ausgezeichneter Forscher und gesuchter Ratgeber}

Herr Strobel hat zahlreiche wissenschaftliche Auszeichnungen erhalten, unter anderem den Adalbert-CzernyPreis der Deutschen Pädiatrischen Gesellschaft 1984 und 2000. Er war Visiting Professor an der Harvard Medical School, Boston, USA, 1989, und Professor des Department of Paediatrics, Postgraduate Institute of Medical Education and Research Chandigarh, Indien, 2001, und er erhielt die Ehrenmitgliedschaft des Royal College of Physicians London, MRCP.

Herr Strobel ist gesuchter Ratgeber zahlreicher Institutionen und Komitees, so etwa der British Postgraduate Medical Federation, 1992-1996, und anderer britischer und europäischer Arbeitsgruppen, insbesondere auf dem Gebiet der Nahrungsmittelallergien und pädiatrisch-gastroenterologischer Fragestellungen. Weiter gehört er dem Lehrkörper des Royal College of Pae-

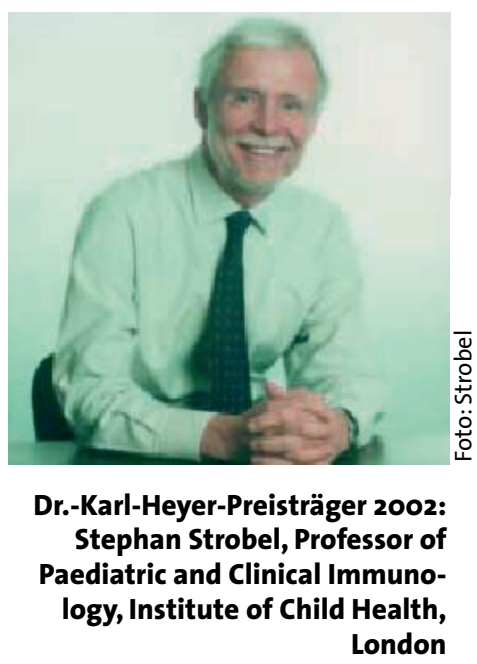

diatrics and Child Health sowie des University College London an. Er ist Mitherausgeber bedeutender wissenschaftlicher Zeitschriften wie Clinical and Experimental Immunology und Paediatric Allergy and Immunology.

\section{Zentrales Interessengebiet Nahrungsmittelallergien}

Hauptthemen seiner vielfach hochrangig geförderten Forschung sind Nahrungsmittelallergien sowie die Immunologie des Gastrointestinaltraktes im Kindesalter. Die wichtigsten Untersuchungen sind der darmassoziierten Immunregulation, den Mechanismen der mukosalen Immuntoleranz, der Wirkung von Allergenen - wie etwa dem Erdnussallergen - auf die Darmschleimhaut und den immunologischen Phänomenen bei der Nahrungsmittelallergie gewidmet.

Herrn Strobels Vita zeigt, dass die Entscheidung für eine berufliche Tätigkeit im angelsächsischen Ausland für den beruflichen Erfolg entscheidend sein kann - und dies nicht erst in jüngster Zeit.

Die DGAI gratuliert Herrn Prof. Strobel zur Verleihung des Dr.-KarlHeyer-Preises 2002 und wünscht ihm weiterhin das notwendige Gespür für wichtige wissenschaftliche Konzepte, Erfolg in seinem akademischen Leben und Glück im privaten Alltag.

Prof. Dr. Gerhard Schultze-Werninghaus, Bochum 